\title{
Healthcare practitioners' views about early hearing detection and intervention practices in KwaZulu-Natal, South Africa
}

\author{
N B Khan, MPH; L Joseph, PhD \\ Discipline of Audiology, College of Health Sciences, University of KwaZulu-Natal, Durban, South Africa \\ Corresponding author: N B Khan (khanna@ukzn.ac.za)
}

\begin{abstract}
Background. Healthcare practitioners' views, understanding of and support for early hearing detection and intervention (EHDI) is essential for its effective implementation

Objectives. To determine public healthcare practitioners' views about EHDI at primary healthcare level based on primary-care nurse's awareness and referral practices related to high-risk factors for hearing loss and the challenges with EHDI implementation.

Methods. A customised questionnaire $(\alpha=0.71)$ was used to survey a sample $(N=38)$ of relevant healthcare practitioners, including audiologists, speech therapists and ear, nose and throat (ENT) specialists from 11 purposively selected hospitals in KwaZulu-Natal, South Africa (SA).

Results. Practitioners had a positive view towards hearing screening being mandatory and that it should form part of the birth package offered to mothers. Approximately $58 \%$ of participants agreed that the list of high-risk factors issued by the Joint Committee on Infant Hearing (JCIH) is relevant in the SA context and 50\% agreed that infants with hearing loss globally present with similar high-risk factors. Approximately $21 \%$ of the participants disagreed with this, suggesting that the risk factors differ among populations. Only $45 \%$ of participants considered primary-care nurses to be aware of high-risk factors for hearing loss. Most were thus unlikely to refer infants for further hearing testing.

Conclusion. Poor awareness, lack of infrastructure for screening and limited resources necessitate further education and training for nurses on risk factors and resource availability at the primary healthcare level is crucial, considering that the majority of South Africans access services at this level of care.
\end{abstract}

S Afr J Child Health 2020;14(4):200-207. https://doi.org/10.7196/SAJCH.2020.v14i4.1708

Hearing loss is the most commonly occurring congenital disability in developing countries and is referred to as a silent epidemic owing to its invisible nature. ${ }^{[1,2]}$ This hinders detection through routine clinical procedures such as well-baby immunisation checks. ${ }^{[1,2]}$ Research suggests that infants with hearing impairment who receive intervention in the first 6 months of their lives, as opposed to those in whom hearing impairment is identified later, are more likely to have speech, linguistic and cognitive development similar to normal-hearing peers. ${ }^{[3]}$ The adverse effects of late detection, such as delayed speech, language and academic development, are extensively reported and contrast with the established benefits of early hearing detection and intervention (EHDI) programmes. ${ }^{[3,4]}$

The aim of an EHDI programme is to identify hearing impairment in infants and provide appropriate early intervention. Early detection and intervention practices begin with newborn hearing screening (NHS), which involves screening an infant either before discharge from hospital or before the age of 3 months at a clinic visit, preferably by means of objective tests such as automated auditory brainstem response (AABR) or otoacoustic emissions (OAE) ${ }^{[5]}$ The importance of EHDI services has increasingly been recognised over the years, resulting in more funding being allocated - mainly in developed countries but also in some developing countries - to reach an increasing number of affected children. Although the EHDI principles are contextually straightforward, the process of screening, diagnosis and intervention is complex. ${ }^{[6]}$
An EHDI programme provides a continuum of care, with a multidisciplinary team of healthcare professionals working together to implement a comprehensive programme that includes NHS, diagnosis, family-centred care and early intervention. ${ }^{[2,3,5,7-9]}$ This team should include family members, audiologists, speech-language therapists, paediatricians, ear, nose and throat (ENT) specialists, nurses and community workers. ${ }^{[10,11]}$ Efforts to implement EHDI programmes should involve engaging professionals in the field, ${ }^{[4,12]}$ as the lack of cohesive teamwork is one of the factors that can hamper effective implementation. ${ }^{[13]}$ Timely intervention and high-quality service are needed for infants to realise their full potential ${ }^{[8]}$ and each healthcare professional in the team has an independent yet cohesive role in managing affected children and providing support to them and their families. ${ }^{[13]}$ Audiologists are trained to detect, diagnose and intervene in such circumstances and are a source of information for parents. ${ }^{[13]}$ ENT specialists have knowledge of ear anatomy, auditory physiology and pathophysiology of hearing loss and can treat childhood hearing conditions, perform surgery and work with audiologists, particularly when a patient needs medical clearance for amplification, an auditory prosthesis or a cochlear implant. ${ }^{[14]}$ Speechlanguage therapists assist with aural rehabilitation in children with hearing loss, including varied communication options, educational placement and regular communication intervention. ${ }^{[6,8]}$

As all the team members have an integral role in an EHDI programme, their views and opinions about the process are important to shape its implementation. Their opinions and professional input 
also influence parents' views and compliance with hearing screening and can affect their seeking further audiology services. It is essential that parents and caregivers receive appropriate information and have timely access to support services to ensure that they are not lost to follow-up, specifically in the case of NHS and EHDI programmes, which greatly affect the uptake of recommended services. ${ }^{[9]}$ Poor return rates during follow-up and a lack of awareness from parents and caregivers can have a detrimental effect on the development of an infant with hearing loss. ${ }^{[9]}$

Adhering to a standard protocol may also improve the quality of screening programmes. ${ }^{[13]}$ In Brazil, for example, the speech therapist and nurse have to guide families towards early diagnosis and intervention and encourage compliance with follow-up testing. ${ }^{[15]}$ In South Africa (SA), there is a shortage of professionals with the necessary skills and qualifications to deliver early intervention services for children with hearing loss. Audiologists are mainly available at hospital level, with limited audiology services available in primary healthcare. ${ }^{[5,16]}$ Nurses at the primary level of care may not necessarily be able to identify hearing loss or associated high-risk factors and make referrals to other levels of the healthcare system.

The dearth of professionals required to deliver EHDI services, especially audiologists, has led to hearing loss being identified late in many children. ${ }^{[2,7,17]}$ In addition, there is documented evidence about the knowledge, attitudes and opinions of doctors and paediatricians about EHDI, ${ }^{[12,15]}$ but very little about that of ENT specialists, audiologists and speech therapists, who are directly involved in managing children with hearing loss. Furthermore, the SA public healthcare sector experiences various challenges in service delivery, including resource constraints exacerbated by poor socioeconomic conditions affecting a large part of the population; these constraints also affect access to audiology services and the provision of EHDI programmes. ${ }^{[18]}$ Various other factors also contribute to infants not being referred to allied healthcare professionals or not having access to screening and early intervention services. ${ }^{[19]}$ Healthcare practitioners' attitudes and lack of knowledge have been implicated as a cause of delayed diagnosis and intervention, with some adopting a wait-and-see approach. ${ }^{[20]}$ A study conducted in the USA by Danhauer et al. ${ }^{[9]}$ concluded that if ENT specialists fail to emphasise the importance of timely audiological follow-up, parents may not take their children for further testing, thus delaying the age of identification and intervention. Johnson et al. ${ }^{[19]}$ found that about a quarter of ENT specialists in their sample incorrectly thought that hearing loss should be identified by 6 months of age and had poor knowledge about certain risk factors (e.g. admission to the neonatal intensive care unit, perinatal hearing loss or late-onset hearing loss).

Some healthcare professionals may disagree with the idea of screening, may not make the necessary referrals and recommendations or may not be aware of the current guidelines regarding EHDI. ${ }^{[19]}$ Healthcare practitioners also have knowledge gaps related to EHDI, causes of hearing loss and referrals and follow-up services: in a study by Ravi et al., ${ }^{[13]}$ some healthcare practitioners even questioned the reliability and cost effectiveness of NHS programmes. In the SA context, healthcare professionals trained to work with hearing impairment are generally not based at the primary level of care and so infants and young children with hearing loss may not be identified early enough unless a referral is received from a nurse at the primary care level. Despite a lack of formal infant screening programmes at clinics, nurses at primary level must at least be able to refer children presenting with high-risk factors for hearing loss. However, a recent study in KwaZulu-Natal, SA, found that primary-care nurses generally refer patients only for risk factors such as otitis media, family history of hearing loss, syndromes and craniofacial anomalies; ${ }^{[21]}$ admission to the neonatal intensive care unit for more than 5 days, hyperbilirubinaemia requiring blood transfusion, meningitis, a low Apgar score, low birthweight, birth asphyxia and exposure to HIV were generally not considered risk factors that warranted referral. ${ }^{[21]}$

For the current study, the risk factors for hearing loss were categorised according to the list of Joint Committee on Infant Hearing (JCIH) risk factors, emerging risks from other developing contexts, known non-JCIH risk factors and risks pertinent to the SA context.

Without collaboration between the various professionals that provide services to infants and children with hearing loss, services can be fragmented, miscommunication can result and misinformation be conveyed. ${ }^{[8]}$ Parents can thus be faced with conflicting advice and information and may be left confused, which can further delay services for their children, ${ }^{[8]}$ and the situation may be aggravated by superstitions or cultural beliefs about the causes of hearing loss.

Parents benefit from the effective collaboration of a team of experts who support and facilitate the early identification of hearing loss and appropriate intervention. In turn, the child benefits from receiving timely and comprehensive intervention to help them reach their full potential. Therefore, understanding healthcare practitioners' opinions, understanding of and support for EHDI is essential for its effective implementation. In this study, we explored the views of public-sector audiologists, speech therapists, speech therapists and audiologists (STAs) and ENT specialists from both rural and urban areas in KwaZulu-Natal on EHDI practices.

\section{Methods}

This study aimed to determine public healthcare practitioners' views about EHDI at primary healthcare level based on high-risk factors for hearing loss with regard to (i) EHDI practices, the contextual relevance of the JCIH criteria and nurses' awareness of high-risk factors for hearing loss; (ii) primary-care nurses' referral practices given both $\mathrm{JCIH}$ and known non-JCIH risk factors for hearing loss; and (iii) challenges and recommendations related to EHDI implementation.

\section{Design}

Data were collected by means of a descriptive survey, with quantitative analysis used to make inferences and learn about a large population by surveying a smaller one.

\section{Study setting and participants}

Audiologists, STAs, ENT specialists and speech therapists from hospitals in urban and rural areas in KwaZulu-Natal were invited to participate in the study. This province has the highest HIV/AIDSassociated and child mortality rates in the country and it is estimated that HIV/AIDS accounts for about half the deaths in under-fives. ${ }^{[22]}$ Other key causes of child mortality in the province include neonatal infections, septicaemia, birth asphyxia and trauma, protein-energy malnutrition, low birthweight, lower respiratory tract infections and diarrhoeal diseases. ${ }^{[22]}$ Some of these conditions are also risk factors for hearing loss in infants.

Eleven public-sector hospitals in the province were purposefully selected for inclusion owing to their employing the targeted healthcare practitioners. On advice from a statistician, all practitioners at the respective hospitals were invited to participate in the study owing to the potentially small target population. Participants were selected for participation on condition that they had at least one year's work experience following the completion of their studies. 
Only 7 of the 11 hospitals (64\%) responded to the request for participation. At the time of the study, these hospitals collectively employed 22 audiologists, 20 speech therapists, 15 STAs, and 22 ENT specialists. Of the total group of 79 potential participants, 5 participated in the pilot study. Of the 74 remaining participants subsequently invited to participate in the main study, 38 agreed, yielding a response rate of $51.3 \%$.

Most of the participants were female $(n=28 ; 73.6 \%)$, with 22 $(57.8 \%)$ between the ages of 26 and 40 years. Audiologists represented the largest group ( $n=17 ; 44.7 \%)$, followed by ENT specialists $(n=8$; $21.1 \%)$, speech therapists $(n=7 ; 18.4 \%)$ and STAs $(n=6 ; 15.8 \%)$. Approximately three-quarters of the participants $(n=28 ; 73.6 \%)$ indicated that English is their first language, whereas six participants (16\%) were isiZulu speakers and four (10.5\%) spoke another language. Close to half of the participants $(n=17 ; 45 \%)$ had more than 5 years' work experience, with the remainder having less than 5 years' experience. Most participants $(n=31 ; 82 \%)$ had a bachelor's degree. Among the remainder, six participants had a master's degree and one a doctoral degree.

Almost all the participants $(n=36 ; 94.7 \%)$ worked at a publicsector health institution, of which about a quarter were in a rural setting. Approximately half of the participants ( $n=17 ; 44.7 \%)$ indicated that NHS screening equipment was available at their workplace, with an equal number indicating that such equipment was not available; four participants were unsure whether the required equipment was available. Twelve particpants (31.6\%) stated that OAE was used and five participants (13.1\%) noted that a combination of OAE/AABR screening was performed at their hospitals; 19 participants (50\%) indicated that other, informal methods were used for screening (e.g. developmental checklists, clinical judgement and parental reports).

Referrals were most commonly received from another healthcare practitioners (e.g. doctors, audiologists or ENT specialists) ( $n=34$; $89.4 \%)$, followed by parents or teachers $(n=29 ; 76.3 \%)$ and nurses (including primary-care nurses) $(n=13 ; 34.2 \%)$. No referrals were noted as being received from traditional healers. The most common reasons for parents seeking assistance related to children's delayed speech and language development $(n=36 ; 81.6 \%)$, poor performance at school $(n=25 ; 65.7 \%)$ or unresponsiveness to speech or sounds $(n=24 ; 63.1 \%)$.

\section{Data collection instrument, procedure and analysis}

The survey required participants to complete a self-administered questionnaire that consisted of four sections. The first section focused on demographic details and the remaining three addressed the stated study objectives, namely $(i)$ healthcare practitioners' opinion related to EHDI practices, the contextual relevance of the JCIH criteria and nurses' awareness of risk factors for hearing loss; (ii) primary-care nurses' referral practices given both JCIH and known non-JCIH risk factors for hearing loss, and (iii) an openended question on challenges with EHDI implementation.

Questionnaire items were derived from studies by Olusanya, ${ }^{[4]}$ Danhauer et al. ${ }^{[9]}$ Arnold et al. ${ }^{[23]}$ Rout and Singh, ${ }^{[24]}$ and Swanepoel et al. ${ }^{[25]}$ The questionnaires were hand delivered to each participating hospital for distribution to the relevant personnel. Participants were asked to complete the questionnaire within 2 weeks of receipt. After the initial period, the hospital was sent a reminder email and the researcher subsequently collected the questionnaires 2 weeks later to allow adequate response time and achieve the maximum response rate. The questionnaire was piloted with five practitioners (two ENT specialists, two audiologists and one speech therapist) at one of the participating hospitals. The findings of the pilot study were not included in the main study, but used to refine the questionnaire ultimately used in the main study.

Internal consistency and reliability of the items in the questionnaire were assessed based on 61 items. Cronbach's alpha was determined to be 0.071 , which indicated acceptable consistency and reliability. ${ }^{[26]}$ To ensure validity, the researcher conducted a comprehensive literature review. The questionnaire was also checked during a pilot process. The anticipated limitations included possible information and selection bias due to non-respondents.

Descriptive and inferential statistics were calculated during data analysis using the Statistical Package for the Social Sciences (SPSS, version 23) (IBM Corp., USA). Descriptive statistics were used to analyse frequency responses and percentage calculations obtained from the closed-ended questions and the Likert scale. The openended question was analysed by drawing on themes and was quantified descriptively. Inferential statistics were used to analyse the relationship between variables. Pearson's $\chi^{2}$ test was used to identify possible associations between demographic variables and participants' expressed views. The statistical significance level was set at $p=0.05$.

\section{Ethical considerations}

An information sheet and consent form were provided to participants prior to their completing the questionnaire. Ethical approval for the study was granted by the Biomedical Research Ethics Committee of the University of KwaZulu-Natal (ref. no. 395/14). Participation was voluntary and participants could withdraw their participation at any stage of the study. Research codes were used to ensure participant anonymity. The researcher completed an online ethics course prior to the study. Permission for the study was also obtained from the provincial Department of Health, district managers and the hospital managers prior to the participants being approached.

\section{Results}

The results are presented in relation to the three study objectives.

\section{Healthcare practitioners' view on practices for early hearing detection and intervention}

Results pertaining to this study objective are presented in Table 1. Almost all participants $(n=36 ; 94.7 \%)$ supported hearing screening for neonates and the majority $(n=30 ; 78.9 \%)$ supported early hearing screening being mandatory. About three-quarters $(n=27 ; 76.3 \%)$ of the participants agreed that auditory screening and care should form part of the birth package. More than two-thirds of the participants $(n=26 ; 68.4 \%)$ strongly agreed that universal NHS warranted healthcare resource expenditure and all participants agreed that identification of hearing loss and subsequent intervention should occur before the age of 6 months.

The majority of the participants $(n=37 ; 94.7 \%)$ indicated AABR or OAE tests to be the best screening options, with 22 (57.9\%) also stating that low-cost screening options at community level may be a feasible option. The majority of participants $(n=27 ; 71.1 \%)$ disagreed with the statement that the current methods for creating awareness of hearing screening and intervention and subsequent caregiver education were effective. A similarly large proportion of participants $(n=29 ; 76.3 \%)$ disagreed with the statement that the referral and follow-up process for infants identified with hearing loss was adequate or effective. Eight participants (21\%) disagreed with the statement that infants with hearing loss will globally present with similar risk factors for hearing loss; $28.9 \%$ of participants $(n=11)$ had a neutral view in response to this item. Almost half of the participants $(n=18 ; 47.4 \%)$ regarded the JCIH list of risk factors 
Table 1. Healthcare practitioners' views on newborn and infant hearing screening $(N=38)$

\begin{tabular}{|c|c|c|c|c|c|c|c|}
\hline Questionnaire item ${ }^{\star}$ & $\begin{array}{l}\text { Strongly } \\
\text { agree, } \%\end{array}$ & $\begin{array}{l}\text { Agree, } \\
\%\end{array}$ & $\begin{array}{l}\text { Neutral, } \\
\%\end{array}$ & $\begin{array}{l}\text { Disagree, } \\
\%\end{array}$ & $\begin{array}{l}\text { Strongly } \\
\text { disagree, \% }\end{array}$ & Mean $(\mathrm{SD})^{\dagger}$ & $p$-value \\
\hline $\begin{array}{l}\text { Hearing and ear care should be part of the birth } \\
\text { package. }\end{array}$ & 76.3 & 21.1 & 2.6 & 0 & 0 & $4.74(0.490)$ & 0.554 \\
\hline All children must be screened at birth. & 60.5 & 34.2 & 2.6 & 2.6 & 0 & $4.53(0.687)$ & 0.197 \\
\hline Hearing screening should be mandatory. & 78.9 & 18.4 & 2.6 & 0 & 0 & $4.76(0.503)$ & 0.452 \\
\hline $\begin{array}{l}\text { UNHS is deserving of healthcare resource } \\
\text { expenditure. }\end{array}$ & 68.4 & 28.9 & 2.6. & 0 & 0 & $4.37(0.589)$ & 0.073 \\
\hline $\begin{array}{l}\text { Hearing loss should be identified early and } \\
\text { intervention commenced before } 6 \text { months of age. }\end{array}$ & 81.6 & 18.4 & 0 & 0 & 0 & $4.82(0.393)$ & 0.465 \\
\hline $\begin{array}{l}\text { Low-cost screening at community level may be a } \\
\text { feasible option. }\end{array}$ & 21.1 & 36.8 & 28.9 & 7.9 & 5.3 & $3.61(1.079)$ & 0.240 \\
\hline $\begin{array}{l}\text { Objective hearing screening using } \mathrm{AABR} \text { and OAE } \\
\text { is the best option for screening. }\end{array}$ & 42.1 & 52.6 & 5.3 & 0 & 0 & $4.66(0.534)$ & 0.409 \\
\hline $\begin{array}{l}\text { Current methods used to educate mothers about } \\
\text { EHDI are appropriate and effective. }\end{array}$ & 0 & 2.6 & 26.3 & 55.3 & 15.8 & $2.16(0.718)$ & 0.573 \\
\hline $\begin{array}{l}\text { The referral and follow-up process is adequate and } \\
\text { effective. }\end{array}$ & 0 & 2.6 & 21.6 & 63.2 & 13.2 & $2.39(0.855)$ & 0.639 \\
\hline $\begin{array}{l}\text { Globally, infants with hearing loss show similar } \\
\text { high-risk factors. }\end{array}$ & 7.9 & 42.1 & 28.9 & 21.1 & 0 & $3.37(0.708)$ & 0.365 \\
\hline $\begin{array}{l}\text { The JCIH list of risk factors is relevant for South } \\
\text { Africa. }\end{array}$ & 10.5 & 47.4 & 39.5 & 2.6 & 0 & $3.66(0.913)$ & 0.438 \\
\hline $\begin{array}{l}\text { Nurses should routinely screen when infants } \\
\text { come in for their immunisation if a high-risk factor } \\
\text { is present. }\end{array}$ & 42.1 & 36.8 & 13.2 & 7.9 & 0 & $4.13(0.935)$ & 0.643 \\
\hline $\begin{array}{l}\text { Primary healthcare nurses are knowledgeable about } \\
\text { the high-risk factors for hearing loss. }\end{array}$ & 2.6 & 2.6 & 39.5 & 42.1 & 13.1 & $2.13(0.665)$ & 0.887 \\
\hline
\end{tabular}

as relevant for the SA context, although 15 (39.5\%) held a neutral view. More than half of the participants $(n=21 ; 55.2 \%)$ disagreed with the statement that nurses were knowledgeable about the highrisk factors for hearing loss; $39.5 \%$ of the participants $(n=15)$ held a neutral view. About $78.9 \%$ of participants $(n=30)$ agreed that nurses should routinely screen for hearing loss at immunisation visits. Pearson's $\chi^{2}$ test did not reveal any statistically significant associations between profession group and the view regarding EHDI practices, with $p>0.05$ in all cases.

In the demographic section, participants were additionally asked if they had screening equipment to conduct hearing screening. Only 17 participants $(44.7 \%)$ indicated that such equipment was available; the others stated that they did not have such equipment available or were unsure. There was a statistically significant association between participants' views on EHDI and equipment availability: participants who had screening equipment in place were more likely to strongly agree with mandatory hearing screening ( $p=0.006)$, universal screening at birth $(p=0.023)$ and universal NHS programmes warranting healthcare resource expenditure $(p=0.020)$. However, this group of participants was less likely to strongly agree with auditory care forming part of primary healthcare $(p=0.014)$.

\section{Healthcare practitioners' view on practices for early hearing detection and intervention}

Participants' views regarding the referral behaviour of primarycare nurses were explored in the context of a list of predefined risk factors, either as stated by the JCIH (2007) (Table 2) or based on known non-JCIH risk factors, factors emerging from developing countries or those identified as relevant to the SA context (Table 3).

Participants indicated that primary-care nurses would probably refer a child for hearing screening based on caregiver concern for speech and language development in $66 \%$ of cases and in $60 \%$ of cases if a craniofacial anomaly was evident. Referrals related to meningitis were likely to be received in only $40 \%$ of cases, with even fewer referrals related to syndromes (37\%), maternal infections (34\%), jaundice $(32 \%)$, family history $(29 \%)$ or prior admission to a neonatal intensive care unit for more than 5 days (29\%). Neurodegenerative disorders, head trauma and chemotherapy were least often the reason for referrals from primary-care nurses.

Participants indicated that recurrent otitis media was the most common reason for referral from primary-care nurses. Low Apgar scores were cited as the reason for referral by less than a third of the participants and asphyxia at birth resulted in referral only in about a quarter of cases. HIV exposure or low birthweight was cited as a referral reason in less than $25 \%$ of cases. Limited referrals were noted for non-JCIH risk factors such as maternal hypertension, birth by an unskilled attendant, undernutrition, consanguinity, sickle cell anaemia, hypoglycaemia and maternal substance abuse.

\section{Challenges and recommendations related to implementation of an early hearing detection and intervention programme}

Most of the participants $(n=29 ; 76 \%)$ agreed that knowledge of hearing and hearing loss is generally poor at primary healthcare 
Table 2. Healthcare practitioners' views $(N=38)$ about referrals received from primary-care nurses based on the JCIH (2007) list of high-risk factors

\begin{tabular}{llll}
\hline Risk factor ${ }^{*}$ & Yes, \% & No, \% & Maybe, \% \\
\hline Caregiver concern & 66 & 8 & 26 \\
Family history & 29 & 29 & 42 \\
Admission to neonatal intensive & 29 & 47 & 24 \\
care unit $>5$ days & & & \\
Maternal infections & 34 & 32 & 34 \\
Hyperbilirubinaemia & 32 & 42 & 26 \\
Craniofacial anomalies & 60 & 10 & 30 \\
Syndromes & 37 & 26 & 37 \\
Neurodegenerative disorders & 11 & 47 & 42 \\
Meningitis & 40 & 34 & 26 \\
Head trauma & 13 & 47 & 40 \\
Chemotherapy & 21 & 53 & 26 \\
& & & \\
JCIH = Joint Commission on Infant Hearing. & \\
${ }^{*}$ Risk factor items were adapted from the JCIH position statement (2007).[31]
\end{tabular}

Table 3. Healthcare practitioners' views $(N=38)$ about referrals from primary-care nurses based on other known non-JCIH high-risk factors

\begin{tabular}{|c|c|c|c|}
\hline Risk factors ${ }^{*}$ & Yes, \% & No, $\%$ & Maybe, \% \\
\hline \multicolumn{4}{|c|}{ Emerging risks from other developing contexts } \\
\hline Maternal hypertension & 5 & 76 & 19 \\
\hline Non-elective caesarean section & 0 & 79 & 21 \\
\hline Unskilled birth attendant & 3 & 79 & 18 \\
\hline Undernutrition & 5 & 74 & 21 \\
\hline \multicolumn{4}{|l|}{ Known non-JCIH risks } \\
\hline Consanguinity & 3 & 60 & 37 \\
\hline Sickle cell anaemia & 2 & 74 & 24 \\
\hline \multicolumn{4}{|l|}{ SA-specific risks } \\
\hline Low Apgar score & 29 & 34 & 37 \\
\hline Recurrent otitis media & 69 & 5 & 26 \\
\hline Low birthweight & 21 & 53 & 26 \\
\hline Birth asphyxia/hypoxia & 26 & 48 & 26 \\
\hline Hypoglycaemia/hyperglycaemia & 8 & 66 & 26 \\
\hline Maternal substance/alcohol abuse & 10 & 74 & 16 \\
\hline HIV exposure & 21 & 47 & 32 \\
\hline \multicolumn{4}{|c|}{$\begin{array}{l}\text { JCIH = Joint Commission on Infant Hearing; SA = South Africa. } \\
{ }^{\star T h e ~ l i s t ~ o f ~ r i s k ~ f a c t o r s ~ w a s ~ c o m p i l e d ~ b a s e d ~ o n ~ q u e s t i o n s, ~ i d e a s ~ a n d ~ i t e m s ~} \\
\text { included in studies by Olusanya }{ }^{[4]} \text { and Swanepoel } \text { et al. } .^{[25]}\end{array}$} \\
\hline
\end{tabular}

level. Other limiting factors for implementing EHDI programmes were cited as shortage of staff $(n=25 ; 66 \%)$ and lack of adequate equipment or poor infrastructure $(n=33 ; 87 \%)$; close to threequarters of participants $(n=28 ; 73 \%)$ referred to a lack of diagnostic audiology services even when hearing loss was identified. A large proportion of participants $(n=23 ; 60 \%)$ stated that infants are not brought in for screening and follow-up services.

Participants were presented with a list of some commonly held cultural beliefs highlighted in other studies and asked whether they were aware of any such cultural beliefs that could hinder access to EHDI services. Very few participants $(n=3 ; 7.8 \%)$ indicated that communities would attribute hearing loss to blood impurities, although 16 participants $(42.1 \%)$ noted that bewitchment and angry ancestors were commonly held beliefs by the community about the causes of hearing loss. Some participants $(n=5 ; 13.1 \%)$ noted
Table 4. Challenges and recommendations related to implementation of early hearing detection and intervention programmes $(N=24)$

\begin{tabular}{|c|c|}
\hline Challenges & $n(\%)$ \\
\hline Poor awareness by nurses about audiology and hearing loss & $8(33)$ \\
\hline $\begin{array}{l}\text { Lack of human, financial and equipment resources (nurses } \\
\text { and audiologists; long waiting lists; poor priority given to } \\
\text { audiology - hospital) }\end{array}$ & $8(33)$ \\
\hline $\begin{array}{l}\text { Lack of awareness among parents and doctors - reasons } \\
\text { for referrals }\end{array}$ & $6(25)$ \\
\hline $\begin{array}{l}\text { Parents do not keep appointments and do not follow up } \\
\text { for further testing }\end{array}$ & $6(25)$ \\
\hline Lack of training by nurses on hearing screening methods & $5(21)$ \\
\hline Poor parental and caregiver education and information & $5(21)$ \\
\hline Different referral protocols & $3(13)$ \\
\hline Lack of screening guidelines & $3(13)$ \\
\hline Stigma associated with disabilities such as hearing loss & $3(13)$ \\
\hline $\begin{array}{l}\text { Late identification even with children presenting with } \\
\text { high risk (developed contexts syndromes are picked } \\
\text { up earlier; risks may differ in developed v. developing } \\
\text { contexts; added risks due to HIV/tuberculosis) }\end{array}$ & $3(13)$ \\
\hline $\begin{array}{l}\text { Language barriers (especially if client not English or } \\
\text { isiZulu speaker) }\end{array}$ & $2(8)$ \\
\hline Staffing constraints and nurses' workloads & $2(8)$ \\
\hline $\begin{array}{l}\text { Practitioners' lack of awareness about risk factors for } \\
\text { hearing loss }\end{array}$ & $2(8)$ \\
\hline Lack of teamwork and collaboration among practitioners & $2(8)$ \\
\hline Lack of transport & $1(4)$ \\
\hline Lack of facilities for cochlear implants & $1(4)$ \\
\hline \multicolumn{2}{|l|}{ Recommendations } \\
\hline $\begin{array}{l}\text { Education and training of nurses and other healthcare } \\
\text { practitioners }\end{array}$ & $8(33)$ \\
\hline $\begin{array}{l}\text { Development and availability of guidelines and resources } \\
\text { for screening }\end{array}$ & $5(21)$ \\
\hline Risk factor identification and training & $3(13)$ \\
\hline $\begin{array}{l}\text { Parent education and awareness to facilitate early } \\
\text { identification }\end{array}$ & $3(13)$ \\
\hline
\end{tabular}

that hearing loss may be attributed to a person's inner spirit being disturbed in some communities, although $34.2 \%$ of participants $(n=13)$ did not feel that communities held any such cultural beliefs about the causes of hearing loss.

Additional challenges and recommendations were noted by 24 participants (63.1\%) in response to an open-ended question (Table 4). Some of these participants provided multifaceted responses, although in some cases no recommendation was offered for a cited challenge.

\section{Discussion}

Hearing loss should be identified early to ensure that children benefit from early intervention to improve hearing outcomes. ${ }^{[8,27]}$ There was an overwhelming agreement among participants in our study that newborns should be screened at birth, that hearing screening should be mandatory and that universal NHS warrants healthcare resource expenditure. Similar findings were reported from the USA by Muñoz and Blaiser. ${ }^{[8]}$ Moeller et al. ${ }^{[12]}$ found related views on hearing screening among physicians as seen in the current study, but noted that concerns about practical and operational issues were also raised. One of the reasons for universal NHS programmes not routinely being implemented relates to their 
omission from birth packages, institutional policies and routine neonatal care. ${ }^{[5,28]}$ In contrast, a USA study showed that about twothirds of the paediatricians surveyed did not support universal $\mathrm{NHS}^{[29]}$ and another study raised concerns about the financial and emotional costs. ${ }^{[12]}$ These reservations can be partly attributed to a large number of false-positive results. ${ }^{[29]}$ Owing to the competing priorities presented by life-threatening conditions such as TB and HIV/AIDS, the cost of implementing a universal NHS programme in SA may not be justified or feasible, despite demonstrated success in early identification and intervention of hearing loss. ${ }^{[16]}$

Some form of NHS was available in some of the hospitals surveyed in our study, albeit using different methods. A USA study showed that either OAE or AABR is commonly used for screening, with some facilities using both methods: $42 \%$ of the surveyed institutions screened patients prior to hospital discharge and 58\% used a two-stage screening protocol in which screening was not deemed complete until an outpatient screening had been performed following discharge. ${ }^{[17]}$ Although OAE is a simple screening method and provides rapid results, it offers limited assessment of the auditory system compared with AABR and can be affected by factors such as vernix, middle ear infection and ambient noise. ${ }^{[30]}$ However, $\mathrm{AABR}$ is not routinely used as a screening mechanism owing to it being time consuming and requiring more skill to administer. ${ }^{[29]}$ In addition, protocols are not standardised and views vary on what constitutes an ideal context-specific screening protocol. ${ }^{[29]}$ Although the majority of participants in the current study agreed that AABR or OAE is the best option for objective screening, this technology is currently not available at community level in SA and low-cost screening tools need to be developed to fill the gaps in auditory care for children in developing countries. About $60 \%$ of participants in our study agreed that low-cost screening at community level might be a feasible option.

It was encouraging to note that all participants in our study agreed that hearing loss needs to be identified and management initiated by 6 months of age. This is consistent with the EHDI guidelines of the Health Professions Council of South Africa ${ }^{[10,11]}$ and the position statement of the JCIH. ${ }^{[31]}$ The finding points to practitioners' concern for the early diagnosis of hearing loss owing to the critical contribution to effective speech, language and socialemotional outcomes compared with late diagnosis. ${ }^{[7,23]}$ In a report from the Western Cape, the average age of diagnosis of permanent childhood hearing loss is cited as 2 years, with enrolment to intervention programmes at 2.5 years, ${ }^{[5,16]}$ despite the Western Cape having a relatively well-established infrastructure for EHDI services compared with other provinces in SA. ${ }^{[5]}$ Similar findings were reported from Gauteng: the average age of diagnosis was reported to be 31 months, hearing aid fittings occurred at 39 months and enrolment into early intervention services at 43 months. ${ }^{[5]}$

Most participants in our study did not consider local referral and follow-up processes to be adequate. This is similar to findings by Danhauer et al.,$^{[9]}$ who reported that only $7.5 \%$ of audiologists and ENT specialists considered the referral and follow-up process in the USA to be adequate and effective. When results from hearing screening indicate that further diagnostic testing is required, parents and caregivers must be equipped with proper information and resources. ${ }^{[7]}$ Although more than $90 \%$ of newborns are screened in the USA, almost half are lost to follow-up as their parents or caregivers do not seek further services, as is required to get the hearing status confirmed. ${ }^{[19]}$ The problems are compounded by a lack of comprehensive follow-up protocols. ${ }^{[12]}$ Considerable loss to follow-up can hinder effective EHDI programme delivery. ${ }^{[12]}$ When researchers in Brazil asked parents why they did not do any follow-ups, respondents indicated that they had forgotten about making a follow-up appointment (30\%) or had no knowledge of the fact that they needed to have the hearing status verified (20\%). ${ }^{[15]}$ The authors noted that it is the responsibility of healthcare practitioners to guide and follow up with parents and caregivers, as there is a contradiction between what healthcare practitioners say they tell parents and what parents report hearing from practitioners. ${ }^{[15]}$

In the current study, less than $5 \%$ of participants indicated current methods for educating parents or caregivers about hearing screening to be adequate or effective. This contrasts with findings from a study in the USA, in which $56.5 \%$ of participants considered the methods for educating parents on EHDI or NHS to be adequate or effective. ${ }^{[9]}$ Another study in the USA found that healthcare professionals' communication to parents regarding NHS was generally limited. ${ }^{[23]}$ This suggests that professionals involved in these programmes should educate parents or caregivers about the importance of compliance regarding following up on audiological management. ${ }^{[9]}$ Healthcare professionals should also encourage parents or caregivers to seek audiological intervention, but this does not always happen and could be attributed to negative attitudes towards EHDI programmes or audiologists. ${ }^{[9]}$ However, as shown by Luz et al., ${ }^{[15]}$ parents do not always know which professional conducted the test on their child (62\% of parents in that study did not know) and it is therefore important to encourage dialogue and engagement to exchange knowledge and empower parents and caregivers. Most parents were educated about hearing screening while they were at the hospital, but in a study that explored parent-centred communication with regard to infant hearing screening, Arnold et al. ${ }^{[23]}$ found that although most parents were informed about hearing screening while at the hospital, education before birth is ideal. ${ }^{[23]}$ They also recommended that primary-care providers needed current information about hearing screening, diagnostic testing and early intervention to share with parents at an appropriate time. ${ }^{[23]}$ However, primary-care providers were not sure about the exact nature of the information parents received and some indicated that the information was too complex and contained jargon that parents may not understand. ${ }^{[23]}$ This links to findings from an earlier study in which parents identified a need for information presented as a roadmap or pathway from the process of screening to intervention, stating that multiple sessions may be necessary. ${ }^{[6]}$ The information provided to parents should not only assist them in recognising the importance of the test results but also generate interest in the auditory health of their children. ${ }^{[15]}$ Such suggestions could be used to shape information shared with parents in the SA context, as many participants in the current study mentioned that current education initiatives are not adequate or effective.

About $50 \%$ of participants in the current study either disagreed with or held a neutral view about the statement that infants with hearing loss will globally show similar risk factors; about $48 \%$ agreed that the JCIH list of risk factors is relevant in the SA context and $40 \%$ remained neutral. Olusanya ${ }^{[4]}$ states that support for the JCIH risk factors derives mainly from studies conducted in the USA and notes that although the epidemiological profile of permanent childhood hearing loss is likely to show marked distinctions across various regions of the world, no systematic attempt has been reported in the literature to establish the relevance and appropriateness of JCIH risk factors for developing countries. ${ }^{[4]}$ Although the JCIH revises their risk factors regularly, it should not be considered a 'gold standard' for all countries, as risk factors may vary considerably. ${ }^{[32]}$ Worldwide, there is no consensus on the relative importance of the risk factors that have been used to screen 
infants for hearing loss and it is therefore important that these are regularly refined for specific contexts. The implementation of risk-based screening is a feasible interim approach, ${ }^{[33]}$ with context-specific risk factors needing to be established and profiled for SA, and their performance determined, to ensure appropriate identification of hearing loss in infants. ${ }^{[33]}$

Most participants agreed that nurses should routinely perform hearing screening during immunisation visits, although just over $50 \%$ of the participants disagreed that nurses were knowledgeable about risk factors for hearing loss. It is important that primary-care nurses working in rural contexts - where there is generally limited access to audiology services - are aware of risk factors to facilitate timely identification of hearing loss and appropriate referral.

It was encouraging that participants noted that $66 \%$ of referrals from primary-care nurses were in response to parental concerns about delayed speech and language development, given that identification of hearing loss is a passive process mainly due to parental concern versus nurses initiating and eliciting this information. ${ }^{[5,27]} \mathrm{A}$ study conducted in the USA showed that $95 \%$ of paediatricians would screen for hearing in response to parents' concerns; ${ }^{[29]}$ furthermore, if they established that high-risk factors were evident, they would screen $68 \%$ of the time. ${ }^{[29]}$

Of concern in the current study was that with regard to JCIH risk factors, only about a third of participants mentioned that they received referrals following hyperbilirubinaemia, family history or admission to the neonatal intensive care unit and just slightly more in the case of meningitis, congenital infections and syndromes being present. The relevance of these factors for hearing screening was highlighted in another study from SA, which showed that hyperbilirubinaemia was one of the most prevalent risk factors for hearing loss, with others including syndromes, congenital infection, craniofacial defects and bacterial meningitis. ${ }^{[25]}$

Participants in the current study indicated that referrals from primary-care nurses were unlikely to be based on emerging risk factors or known non-JCIH risk factors. In some countries in sub-Saharan Africa and south-east Asia, known non-JCIH risk factors for hearing loss, such as consanguinity and sickle cell anaemia, are fairly common and related to specific ethnic groups. ${ }^{[4]}$ Emerging risk factors from developing countries (e.g. Nigeria) include maternal hypertensive disorders during pregnancy, non-elective caesarean delivery, an unskilled attendant handling delivery and undernutrition. ${ }^{[4]}$ In the local context, primary-care nurses should be aware of these risk factors.

Most of the participants (69\%) indicated that primary-care nurses refer children for recurrent otitis media. In a study by Biagio et al. ${ }^{[34]}$ regarding paediatric otitis media at a primary healthcare clinic in Johannesburg, SA, the prevalence of chronic suppurative otitis media was $6.6 \%$. Evidence suggests that poorly or unmanaged otitis media can lead to permanent hearing loss. ${ }^{[34]}$ Participants in the current study indicated that a low Apgar score, low birthweight or birth asphyxia could lead to referral from primary-care nurses in about a quarter to a third of cases. Fewer (21\%) noted that a referral was likely if a child was exposed to HIV. Two other studies ${ }^{[18,25]}$ also identified asphyxia, low birthweight and prematurity as risk factors in the SA context and educating primary-care nurses about such risk factors could contribute to the timely identification of hearing loss at community level, especially given the critical shortage of skilled professionals at this level of care and lack of resources to support implementation of a universal NHS programme.

Although $92 \%$ of participants in the current study did not believe that communities attributed hearing loss to blood impurities, a study by Swanepoel and Almec, ${ }^{[35]}$ which investigated maternal knowledge and attitudes towards infant hearing loss in an SA sample, showed that $57 \%$ of respondents held at least one cultural belief regarding hearing loss, with blood impurities cited by $44 \%$ of participants. In the same study, bewitchment was cited by $29 \%$ of participants as a possible cause of hearing loss; this was slightly higher in the current study, with $42 \%$ of participants indicating that they believed communities regard this as a cause of hearing loss. Generally, appropriate health information helps to equip people to access services, which, if not available, result in poor primary and secondary prevention of diseases or health conditions, often aggravated by superstitious beliefs. ${ }^{[35]}$

In the current study, participants provided various reasons for late detection and intervention, including poor follow-up return rates, parents or caregivers having limited understanding of the importance of hearing screening, nurses having limited training and awareness of hearing loss, and a lack of resources. Similar findings were reported in a study by Petrocchi-Bartal and Khoza-Shangase ${ }^{[36]}$ in clinics in Gauteng and North West provinces, where participants did not all have access to equipment owing to budgetary and personnel constraints. The lack of urgency and low priority given to hearing impairment at primary healthcare level may be due to issues related to poverty and the burden of life-threatening diseases such as HIV/AIDS and TB. Given the limited number of audiologists available, primary-care nurses need to be trained in identifying hearing loss, understanding high-risk factors relevant to the SA context, conducting hearing screening using objective methods if available and making appropriate referrals. Parental education about the consequences of unmanaged hearing loss is important and parents or caregivers should be provided with the necessary support to minimise loss to follow-up. Having more resources available, developing guidelines for hearing screening and fostering support for and encouraging teamwork among healthcare practitioners may facilitate better EHDI services.

\section{Study limitations}

Owing to the limited sample size, findings cannot be generalised to other contexts.

\section{Conclusion}

Healthcare practitioners were supportive of EHDI programmes, especially NHS, as most expressed it should be mandatory and form part of the birthing package. Healthcare practitioners in this study considered hearing screening to warrant healthcare resource expenditure and that identification of hearing loss and subsequent intervention should be in place by the age of 6 months already, consistent with the EHDI position statement. Although participants noted AABR and OAE to be the most appropriate objective screening tests, they noted that low-cost screening at community level might be a feasible option in the absence of such tests. Concerns were related to referral practices, follow-up compliance from parents and caregivers, and current methods of educating parents and caregivers. Most participants agreed that nurses should screen children when they attend clinics for their immunisation visits, but more than half of the participants did not consider nurses to be adequately knowledgeable about the risk factors for hearing loss, necessitating education and training in this area. Various challenges to implementation of EHDI programmes were mentioned, such as poor knowledge about hearing loss and associated risk factors at community level, the lack of infrastructure to support screening, and limited resources. Recommendations arising from this study include appropriate education and training for nurses, improving parental education, 
making resources available and identifying contextually relevant risk factors. Healthcare practitioners have a crucial role in the implementation of EHDI programmes and should therefore be involved in advocating for services and resources. They should work closely with primary-care nurses, parents or caregivers and other team members to ensure cohesive execution of an EHDI programme.

Declaration. The research reported here forms part of research requirements for completion of a $\mathrm{PhD}$ degree in Audiology at the University of KwaZulu-Natal.

Acknowledgements. The authors would like to thank all participants in the study.

Author contributions. NBK was the primary investigator and collected the data for the study. LJ was the study supervisor. NBK and LJ both contributed to manuscript development.

Funding. None.

Conflicts of interest. None.

1. Swanepoel D, Hugo R, Louw B. Infant hearing screening at immunization clinics in South Africa. Int J Pediatric Otorhinol 2007;70(7):1241-1249. https:// doi.org/10.1016/j.ijporl.2006.01.002

2. Kanji A, Kara R. Pediatric physicians' referral of children aged 0-3 years for audiological evaluation in the public health care sector. Audiol Res 2013:3(1):e7. https://doi.org/10.4081/audiores.2013.e7

3. Yoshinga-Itano C. Levels of evidence: Universal newborn hearing screening (UNHS) and early hearing detection and intervention systems (EHDI). J Commun Disord 2004;37(5):451-465. https://doi.org/10.1016/j. jcomdis.2004.04.008

4. Olusanya BO. Making targeted screening for infant hearing loss an effective option in less developed countries. Int J Pediatr Otorhinolaryngol 2011;75:316321. https://doi.org/10.1016/j.ijporl.2010.12.002

5. Swanepoel D, Störbeck C, Friedland P. Early hearing detection and intervention in South Africa. Int J Pediatr Otorhinolaryngol 2009;73(6):783-786. https://doi. org/10.1016/j.ijporl.2009.01.007

6. Russ SA, Hanna D, DesGeorges J, Forsman I. Improving follow-up to newborn hearing screening: A learning-collaborative experience. Pediatrics 2010;126(Suppl 1):S59-S69. https://doi.org/10.1542/peds.2010-0354k

7. Houston KT, Muñoz KF, Bradham TS. Professional development: Are we meeting the needs of state EHDI programs? Volta Rev 2011;111(2):209-223.

8. Muñoz K, Blaiser K. Audiologists and speech-language pathologists: Making critical cross-disciplinary connections for quality care in early hearing detection and intervention. Perspect Audiol 2011;7(1):34-42. https://doi. org/10.1044/poa7.1.34

9. Danhauer JL, Johnson CE, Finnegan D, et al. A national survey of pediatric otolaryngologists and early hearing detection and intervention programs. J Am Acad Audiol 2006;17(10):708-721. https://doi.org/10.3766/jaaa.17.10.3

10. Health Professions Council of South Africa. Early hearing detection and intervention: guidelines (2007). http://www.hpcsa.co.za/Uploads/editor/ UserFiles/downloads/speech/early_hearing_detection_statement.pdf (accessed 10 January 2018)

11. Health Professions Council of South Africa. Early hearing detection and intervention: guidelines (2018). https://www.hpcsa.co.za/Uploads/SLH/ Guidelines\%20for\%20Early_Hearing_Detection_and_Intervention (EHDI)_2018.pdf (accessed 19 April 2019).

12. Moeller MP, White KR, Shisler L. Primary care physicians' knowledge, attitudes, and practices related to newborn hearing screening. Pediatrics 2006;118(4):1357-1370. https://doi.org/10.1542/peds.2006-1008

13. Ravi R, Gunjawate DR, Yerraguntla K, Rajashekhar B. Systematic review of knowledge of, attitude towards, and practices for newborn hearing screening among healthcare professionals. Int J Paediatr Otorhinolaryngol 2018;104:138144. https://doi.org/10.1016/j.ijporl.2017.11.004
14. Doyle KJ, Ray RM. The otolaryngologist's role in management of hearing loss in infancy and childhood. Ment Retard Dev Disabil Res Rev2003;9(2):94-102. https://doi.org/10.1002/mrdd.10065

15. Luz I, Ribas A, Kozlowski L, Willig M, Berberian AP. Newborn hearing screening in a public maternity ward in Curitiba, Brazil: Determining factors for not retesting. Int Arch Otorhinolarygol 2016;20(4):300-304. https://doi. org/10.1055/s-0035-1567866

16. Störbeck C, Young A. The HI HOPES data set of deaf children under the age of 6 in South Africa: Maternal suspicion, age of identification and newborn hearing screening. BMC Pediatr 2016;16:45. https://doi.org/10.1186/s12887016-0574-1

17. White KR. The current status of EHDI programs in the United States. Ment Retard Dev Disabil Res Rev 2003;9(2):79-88. https://doi.org/10.1002/mrdd.10063

18. Kanji A, Khoza-Shangase K. The occurrence of high-risk factors for hearing loss in very-low-birth-weight neonates: A retrospective exploratory study of targeted hearing screening. S Afr J Commun Disord 2012;59(1):3-7. https:// doi.org/10.7196/SAJCD.120

19. Johnson CE, Danhauer JL, Granali A, Ross M, Harrison J, Cresawn CS. Systematic review of physicians' knowledge of, participation in, and attitudes toward newborn hearing screening programs. Semin Hear 2009;30(3):149-164. https://doi.org/10.1055/s-0029-1225401

20. Moeller MP, Eiten L, White K, Shisler L. Strategies for educating physicians about newborn hearing screening. J Acad Rehabil Audiol 2006;39:11-32.

21. Khan NB, Joseph L, Adhikari M. The hearing screening experiences and practices of primary health care nurses: Indications for referral based on highrisk factors and community views about hearing loss. Afr J Prim Health Care Fam Med 2018;10(1):1848. https://doi.org/10.4102/phcfm.v10i1.1848

22. Bradshaw D, Nannan N, Laubscher, R, et al. Mortality Estimates for Kwa-Zulu Natal Province, 2000. South African National Burden of Disease Study. https:// www.samrc.ac.za/sites/default/files/files/2017-07-03/kwazulunatal.pdf

23. Arnold CL, Davis TC, Humiston SG, et al. Infant hearing screening: Stakeholder recommendations for parent-centered communication. Pediatrics 2006;117(5 Pt 2):S341-S354. https://doi.org/10.1542/peds.2005-2633N

24. Rout N, Singh U. Age of suspicion, identification and intervention for rural Indian children with hearing loss. Eastern J Med 2010;15(3):97-102.

25. Swanepoel D, Johl L, Pienaar D. Childhood hearing loss and risk profile in a South African population. Int J Pediatr Otorhinolaryngol 2013;77(3):394-398. https://doi.org/10.1016/j.ijporl.2012.11.034

26. George D, Mallery P. SPSS for Windows Step by Step: A Simple Guide and Reference. 11.0 Update. 4th ed. Boston: Allyn \& Bacon, 2002.

27. Swanepoel D. Early detection of infant hearing loss in South Africa. S Afr Med J 2009;99(3):158-159.

28. Meyer ME, Swanepoel D. Newborn hearing screening in the private health care sector - a national survey. S Afr Med J 2011;101(9):665-667.

29. Wall TC, Senicz E, Evans HH, Woolley A, Hardin JM. Hearing screening practices among a national sample of primary care pediatricians. Clin Pediatr 2006;45(6):559-566. https://doi.org/10.1177/0009922806290611

30. Kanji A, Khoza-Shangase K. Feasibility of newborn hearing screening in a public hospital setting in South Africa: A pilot study. S Afr J Comm Dis 2016;63(1):150. https://doi.org/10.4102/sajcd.v63i1.150

31. Joint Committee on Infant Hearing. Year 2007 Position Statement: Principles and Guidelines for Early Hearing Detection and Intervention Programs. Pediatrics 2007;120(4):898-921. https://doi.org/10.1542/peds.2007-2333

32. Korres S, Balatsouras D, Nikolopoulos T, Korres G, Ferekidis E. Making universal newborn hearing screening a success. Int J Pediatr Otorhinolaryngol 2006;70(2):241-246. https://doi.org/10.1016/j.ijporl.2005.06.010

33. Kanji A. Early hearing screening in South Africa - time to get real about context. S Afr J Child Health 2016;10(4):192. https://doi.org/10.7196/SAJCH.2016. v10i4.1298

34. Biagio L, Swanepoel D, Laurent C, Lundberg T. Paediatric otitis media at a primary healthcare clinic in South Africa. S Afr Med J 2014;104(6):431-435. https://doi.org/10.7196/SAMJ.7534

35. Swanepoel D, Almec N. Maternal views on infant hearing loss and early intervention in a South African community. Int J Audiol 2008;47(Suppl 1):S44-S48. https://doi.org/10.1080/14992020802252279

36. Petrocchi-Bartal L, Khoza-Shangase K. Hearing screening procedures and protocols in use at immunisation clinics in South Africa. S Afr J Commun Disord 2014;61(1):66. https://doi.org/10.4102/sajcd.v61i1.66

Accepted 1 June 2020 